\title{
Solvent-dependent Characterization of Fucoxanthin through 2D Electronic Spectroscopy Reveals New Details on the Intramolecular Charge Transfer State Dynamics
}

\author{
Giampaolo Marcolin, ${ }^{1}$ Elisabetta Collini ${ }^{1 *}$ \\ ${ }^{1}$ Department of Chemical Sciences, University of Padova, Via Marzolo 1, I-35131 Padova, Italy.
}

\section{Contents}

S1. 2DES Setup

S2. 2DES Pulse Characterization

S3. Additional 2DES maps

S4. DAS maps for Fucoxanthin in Methanol and Fucoxanthin in Toluene

S5. Additional local fitting results

S6. Beating Analysis 


\section{S1. 2DES Setup}

2DES measurements have been performed with the setup described in ref [1]. Briefly, a Ti:Sapphire laser system ( $3 \mathrm{KHz}$ Coherent Libra) generates output pulses at $800 \mathrm{~nm}$, then converted with a non-collinear optical amplifier (NOPA) (Light Conversion TOPAS White) in broad visible pulses. The central wavelength of the broad-band pulses, set at $555 \mathrm{~nm}$, cannot be pushed further towards blue, due to NOPA limitations. A prism compressor system coupled with a pulse shaper (Fastlite Dazzler) permits to get the transform-limited condition for the pulses at the sample position. The setup adopts the fully non-collinear BOXCARS geometry where the laser pulses are split in four identical beams placed on the vertexes of an imaginary square. Three of the resulting beams (the exciting beams) are delayed temporally by pairs of $4^{\circ} \mathrm{CaF}_{2}$ wedges. One wedge of each pair is mounted onto a motorized stage that regulates the thickness of the medium crossed by the beam, modulating the delay times of the pulses with a temporal resolution of $0.07 \mathrm{fs}$. The fourth beam, instead, is attenuated of 3 orders of magnitude and used as local oscillator (LO) to perform heterodyne detection. The four pulses are then refocused on the sample and, for phase-matching conditions, the signal is emitted on the same direction of the LO pulse. The signal resulting from the interference of the signal and LO is then detected through a spectrograph and an Andor Zyla 5.5 sCMOS camera with 5.5 megapixels.

The pulse duration, optimized through TG-FROG measurements, was compressed to about $10 \mathrm{fs}$ with a spectral bandwidth of about $1470 \mathrm{~cm}^{-1}$. The pulse energy at the sample position was set to $7 \mathrm{~nJ}$ per pulse. The outcome of the 2DES experiment is a 3D array of data describing the evolution of 2D frequency-frequency correlation maps as a function of the population time $t_{2}{ }^{2-4}$ The population time $t_{2}$ was scanned from 0 to $1000 \mathrm{fs}$ with steps of 7.5 fs. Each set of measurements was repeated three times to reduce noise and ensure reproducibility. The data have then been analyzed with the global fitting methodology proposed in Volpato et al.. 5

Fucoxanthin was obtained from Sigma-Aldrich ${ }^{\circledR}$ and dissolved in the following anhydrous solvents: methanol, acetone, and toluene. The concentration of the samples was adjusted so to reach an absorbance of $\sim 0.25$ in a $1 \mathrm{~mm}$ cuvette at $520 \mathrm{~nm}$, leading to a concentration of $0.8 \mathrm{mM}$. The presence of aggregates in these conditions has been excluded verifying that the absorption spectrum does not significantly vary in a range of concentrations going from $1 \mu \mathrm{M}$ to $1 \mathrm{mM}$. For each sample, steady-state absorption spectra were acquired before and after each 2DES experiment to verify the absence of degradation of the sample during the measurements. 


\section{S2. 2DES Pulse Characterization}
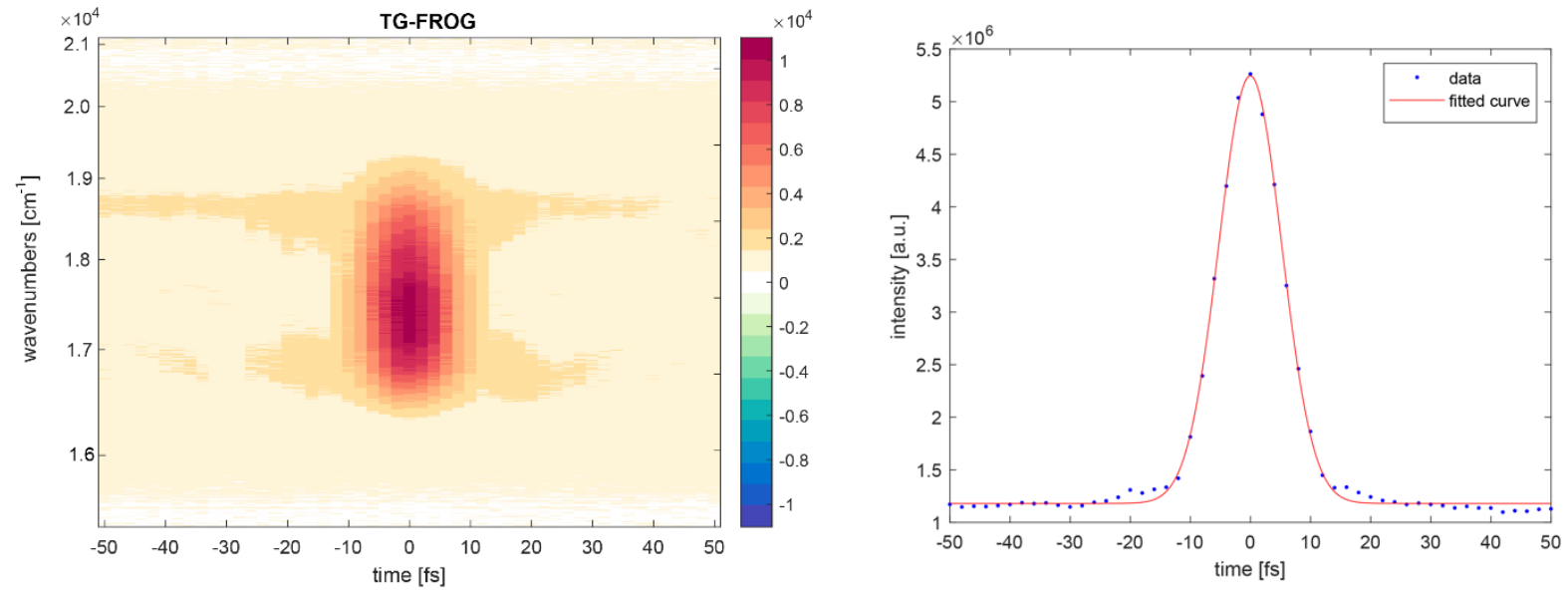

Figure S1. Characterization of the pulse duration through frequency resolved optical gating (FROG) measurements performed on a solvent in the same experimental conditions of the 2DES experiments. (left) FROG plot; (right) Gaussian fit of the FROG signal integrated along the frequency dimension to estimate the pulse duration $(8.7 \mathrm{fs})$. 


\section{S3. Additional 2DES maps}

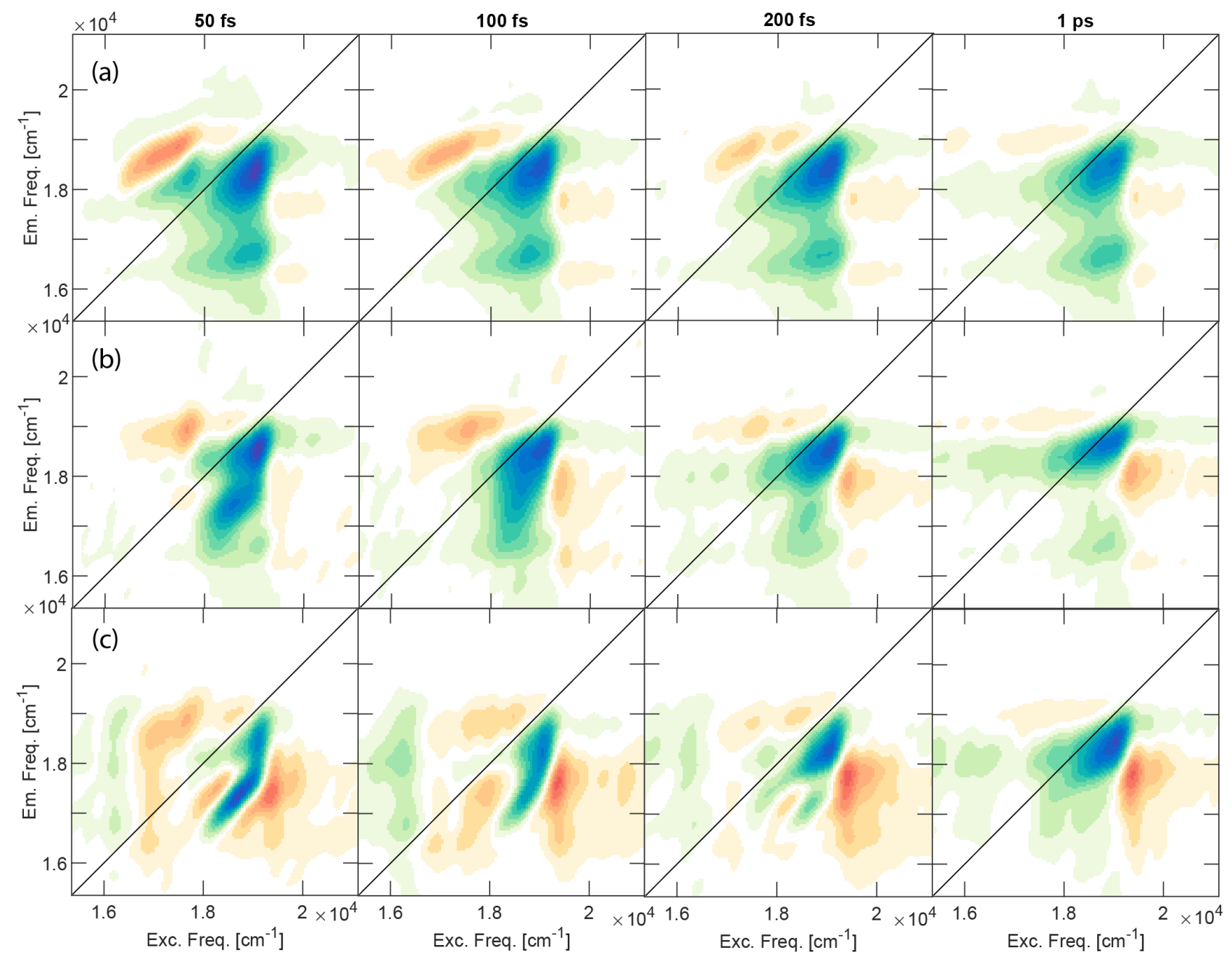

Figure S2. 2DES Rephasing maps of fucoxanthin dissolved in a) methanol; b) acetone; c) toluene. 


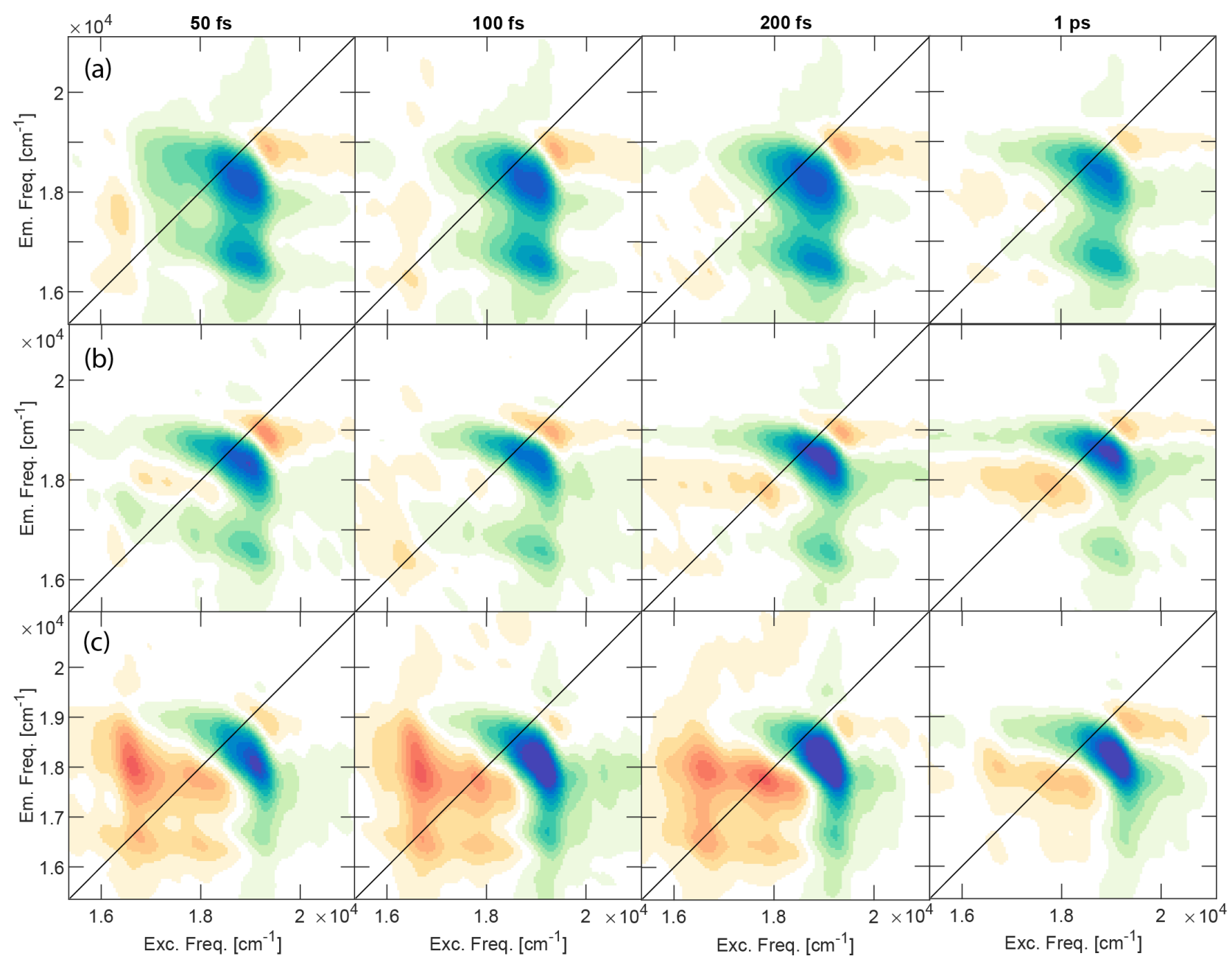

Figure S3. 2DES Non-Rephasing maps of fucoxanthin dissolved in a) methanol; b) acetone; c) toluene. 
S4. 2D-DAS maps for Fucoxanthin in Methanol and Toluene
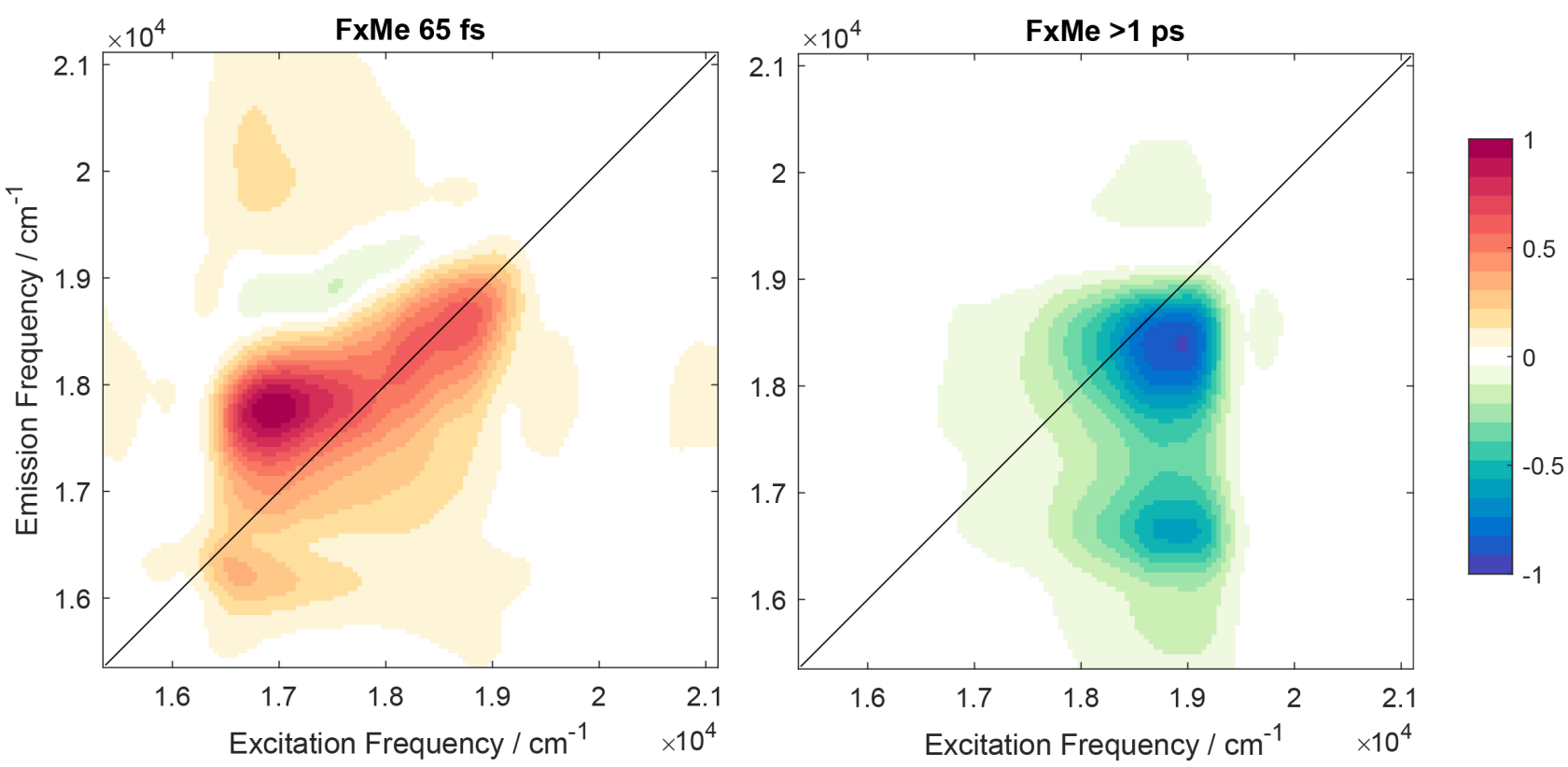

Figure S4. 2D-DAS maps of Fucoxanthin in Methanol
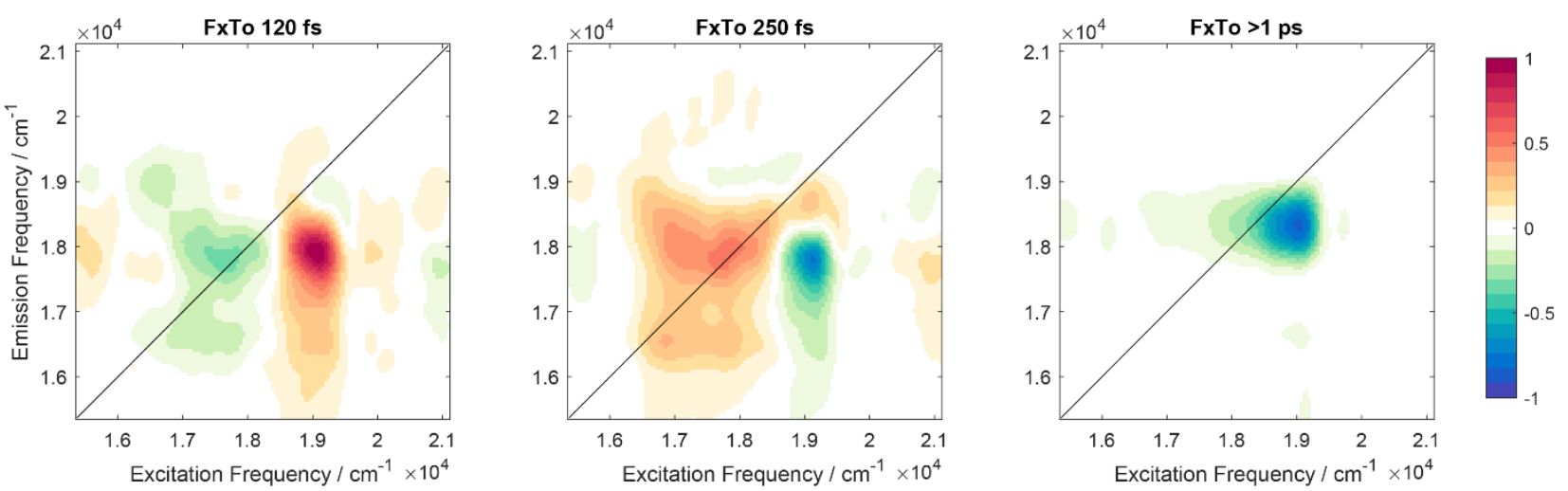

Figure S5. 2D-DAS maps of Fucoxanthin in Toluene 


\section{S5. Additional local fittings results}

Local fittings based on a 3-exponential model have been performed at specific coordinates to untangle the multiple dynamics taking place in hundreds fs time scale.

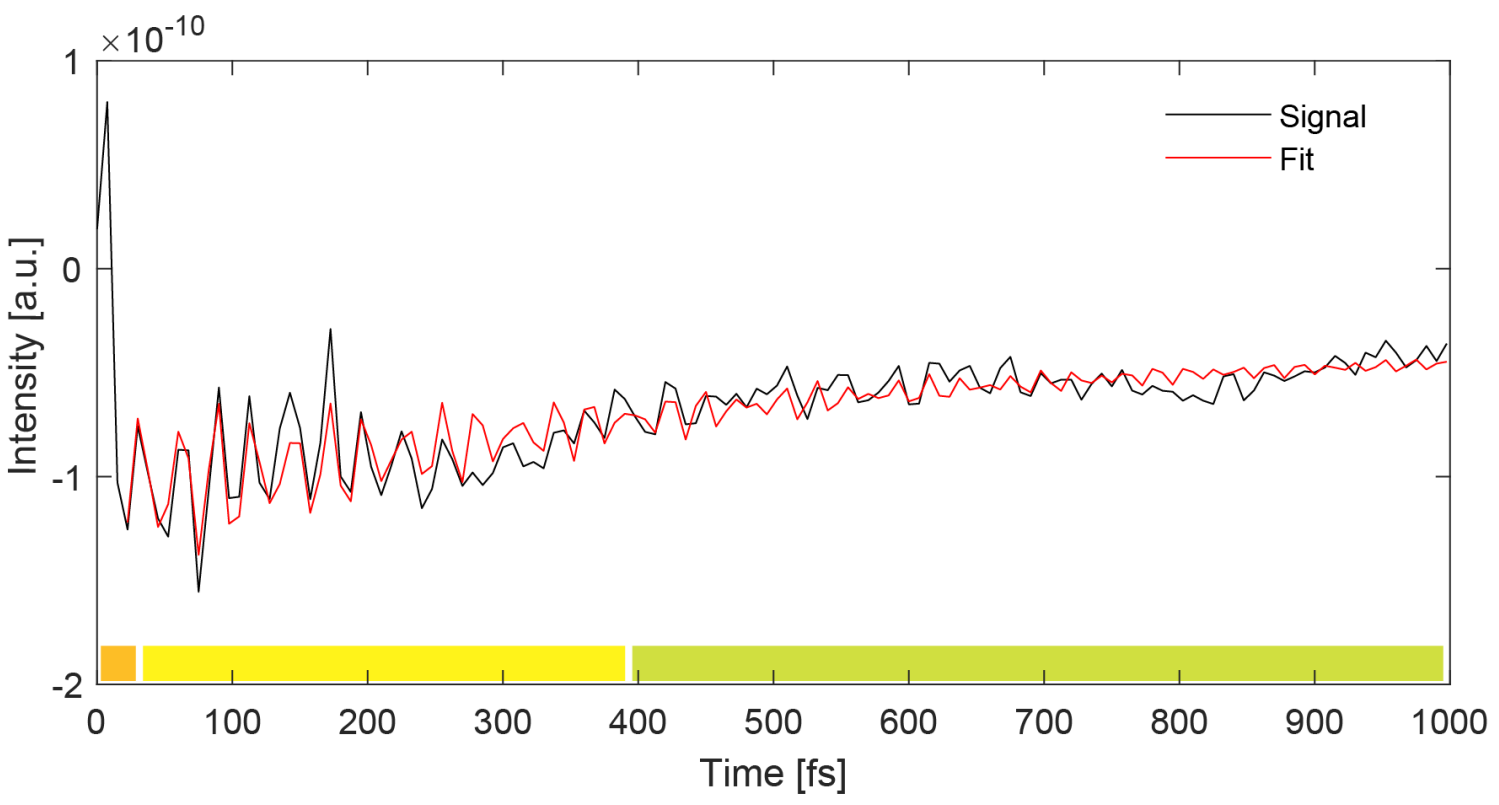

Figure S6. Decay trace showing the $t_{2}$ evolution of the signal at the coordinates $19000,17800 \mathrm{~cm}^{-1}$ for the FxAc sample. The three colored bars indicate the time ranges for the three processes that govern this evolution: the rising of the signal associated to the $\mathrm{S}_{2} \rightarrow$ hot $\mathrm{S}_{1}$ internal conversion (orange), the fast decay associated to the hot $S_{1}$ relaxation (yellow), and the slow decay as the population is restored to the ground state (green). The corresponding time constants are: $<10 \mathrm{fs}, 310 \mathrm{fs}$ and $>1 \mathrm{ps}$, respectively

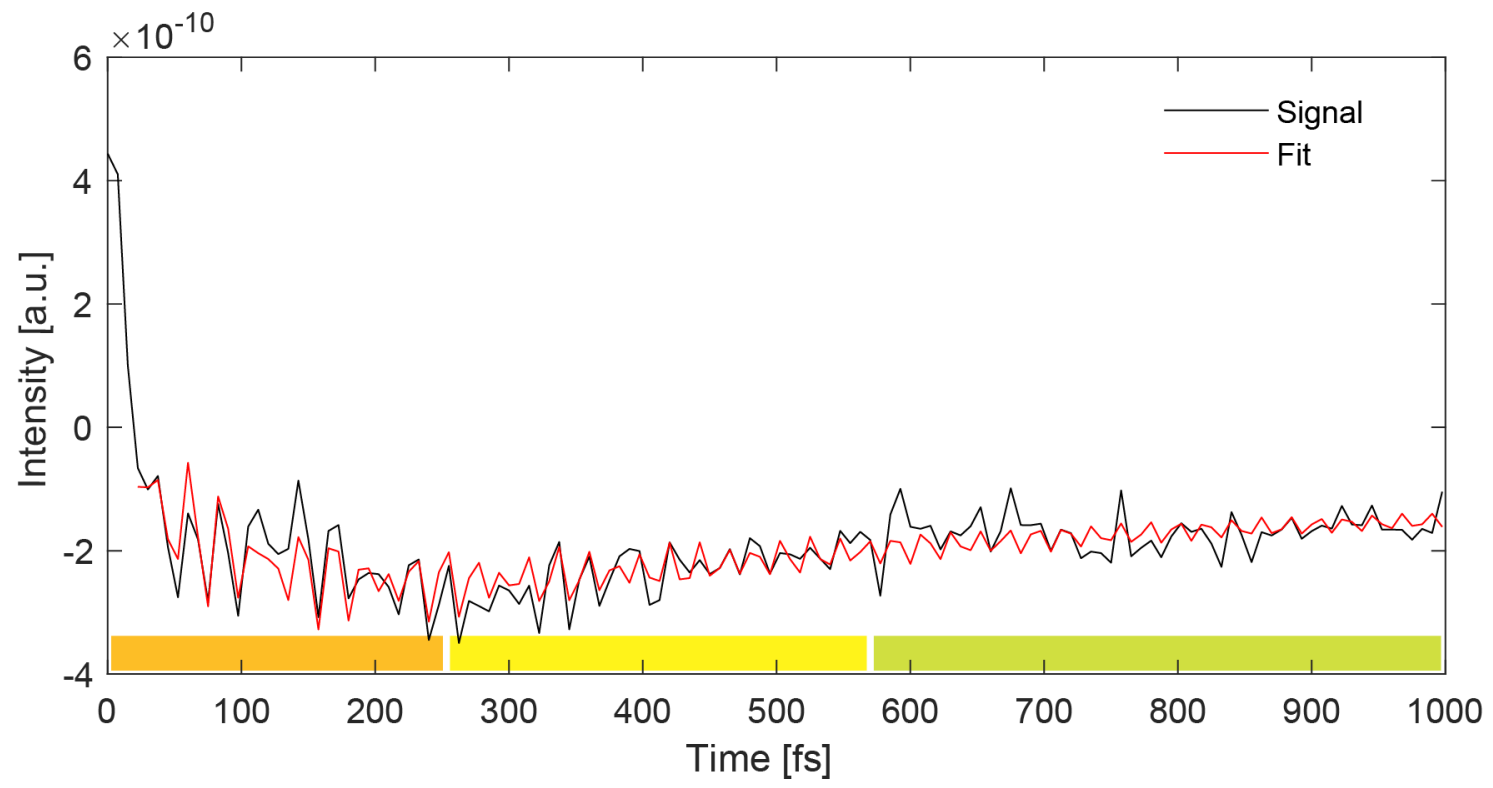

Figure S7. Same as Figure S6 for FuTo sample. The corresponding time constants are: $120 \mathrm{fs}, 310 \mathrm{fs}$ and $>1$ ps. 


\section{S6. Beating Analysis}

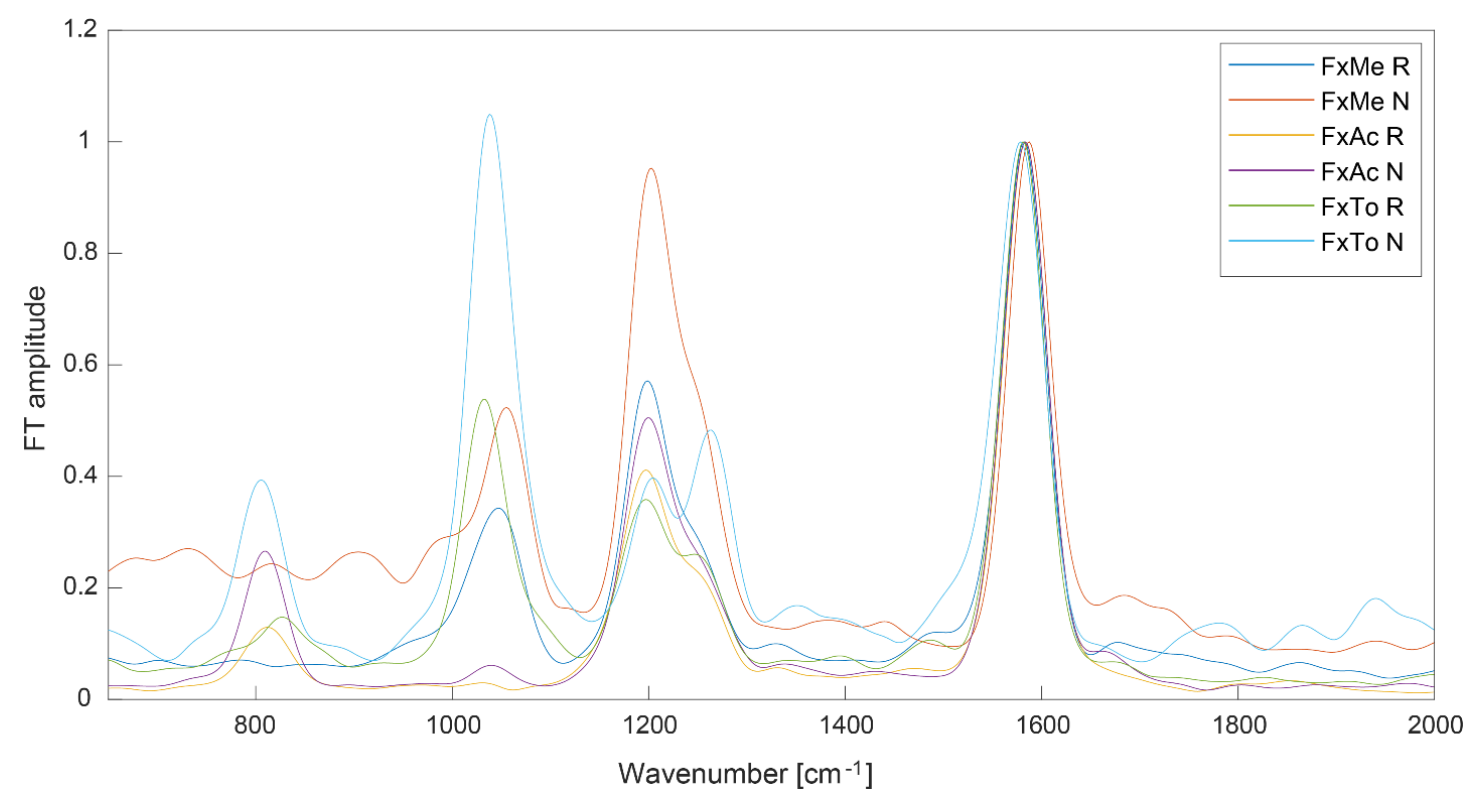

Figure S8. Fourier spectrum analysis of the beatings in the $t_{2}$ evolution of the signal for the three samples ( $\mathrm{R}=$ rephasing, $\mathrm{N}=$ non rephasing). The main beating components correspond to vibrational modes of $\mathrm{Fx}$ $\left(\sim 1200, \sim 1280, \sim 1580 \mathrm{~cm}^{-1}\right) .{ }^{6,7}$ Signals below $1200 \mathrm{~cm}^{-1}$ are due to non-resonant solvent contributions: the main peak of methanol is centered at $\sim 1050 \mathrm{~cm}^{-1}$ and the one of acetone at $\sim 800 \mathrm{~cm}^{-1}$. Toluene, instead, has two main components at $\sim 800 \mathrm{~cm}^{-1}$ and $\sim 1030 \mathrm{~cm}^{-1}$.

\section{References}

(1) Bolzonello, L.; Volpato, A.; Meneghin, E.; Collini, E. Versatile Setup for High-Quality Rephasing, Non-Rephasing, and Double Quantum 2D Electronic Spectroscopy. J. Opt. Soc. Am. B 2017, 34 (6), 1223-1233. https://doi.org/10.1364/josab.34.001223.

(2) Bránczyk, A. M.; Turner, D. B.; Scholes, G. D. Crossing Disciplines - A View on Two-Dimensional Optical Spectroscopy. Ann. Phys. (Berlin) 2014, 526 (1-2), 31-49. https://doi.org/10.1002/andp.201300153.

(3) Collini, E. Spectroscopic Signatures of Quantum-Coherent Energy Transfer. Chem. Soc. Rev. 2013, 42 (12), 4932-4947. https://doi.org/10.1039/c3cs35444j.

(4) Gelzinis, A.; Augulis, R.; Butkus, V.; Robert, B.; Valkunas, L. Two-Dimensional Spectroscopy for Non-Specialists. Biochim. Biophys. Acta 2019, 1860 (4), 271-285. https://doi.org/10.1016/j.bbabio.2018.12.006.

(5) Volpato, A.; Bolzonello, L.; Meneghin, E.; Collini, E. Global Analysis of Coherence and Population Dynamics in 2D Electronic Spectroscopy. Opt. Express 2016, 24 (21), 24773. https://doi.org/10.1364/oe.24.024773.

(6) Rimai, L.; Heyde, M. E.; Gill, D. Vibrational Spectra of Some Carotenoids and Related Linear Polyenes. A Raman Spectroscopic Study. J. Am. Chem. Soc. 1973, 95 (14), 4493-4501.

(7) Merlin, J. C. Resonance Raman Spectroscopy of Carotenoids and Carotenoid-Containing Systems. Pure Appl. Chem. 1985, 57 (5), 785-792. 